pump signal and applying a new trigger pulse with the opposite phase.

The piezoelectric approach allows the tuning, pumping ${ }^{5}$ and read-out mechanisms to all be integrated in the mechanical beam ${ }^{4}$. Maintaining the efficiency of these mechanisms as devices are scaled to smaller and smaller sizes is a crucial issue in all NEMS work ${ }^{6}$, and the NTT prototypes show promise in this respect because the mechanisms are all integrated into the devices. Indeed, tantalizingly little power is dissipated in nanomechanical motions, particularly as devices are scaled down in size, so the challenge will be to find a transduction technique for converting electrical energy into mechanical energy and back that can take advantage of these low power levels without any losses in sensitivity.
High-speed operation (at gigahertz frequencies) is also inherently possible in the NEMS domain, although this leads to other challenges that will need to be overcome. Finally, many parametrons must work in concert to perform computations. In the original LC approach, the oscillators were connected by transformers to make complete logic circuits and prototype general-purpose computers. Engineering of the mechanical coupling between the new parametrons has yet to be demonstrated, but it has been studied for nanomechanical resonators in other contexts ${ }^{7}$.

This work is, in part, the resumption of a noble tradition. Mechanical measurements evolved to exquisite sensitivity before electronics-based instrumentation became standard. Now, mechanical devices that are spin-offs from research in the semiconductor industry are creating new possibilities for largescale integrated mechanical computing. It is too early to say what form a practical nanomechanical computer might take, or if it will ever prevail as a viable computing alternative, but the parametron presents researchers with another building block in their efforts to develop mechanical logic devices.

\section{References}

1. Roukes, M. L. IEEE IEDM Technical Digest 539-542 (2004).

2. Mahboob, I. \& Yamaguchi, H. Nature Nanotech. 3 , 275-279 (2008)

3. Goto, E. Proc. Inst. Radio Eng. 47, 1304-1316 (1959).

4. Mahboob, I. \& Yamaguchi, H. Appl. Phys. Lett. (in the press)

5. Masmanidis, S. C. et al. Science 317, 780-783 (2007).

6. Ekinci, K. L. Small 1, 786-797 (2005).

7. Zalalutdinov, M. K. et al. Appl. Phys. Lett. 88, 143504 (2006).

\title{
QUANTUM INFORMATION
}

\section{Fraction stations}

Single-electron transistors might appear to be the ultimate in nanoelectronic devices, but it has been known for about a decade that quasiparticles with fractional charges, such as one-third and one-fifth of the electron charge, can be found in semiconductor structures under extreme conditions. Now, two independent groups of physicists have found evidence for quasiparticles with one-quarter of the electron charge. In addition to opening up a new frontier for research into the fractional quantum Hall effect, such particles could one day be exploited in a topological quantum computer that is much less prone to errors than other approaches to quantum computation.

Condensed-matter physicists use the name quasiparticle to describe a lowenergy excitation in a system of interacting electrons. These quasiparticles can move through the system like a conventional particle, and most of them have the same charge as the electron, $e$. However, when electrons are confined to move in two dimensions in strong magnetic fields at cryogenic temperatures, they can form new quasiparticles with fractional charges that are part electron and part magnetic field line.

Merav Dolev and colleagues at the Weizmann Institute of Science in Israel and, independently, a team from MIT, Harvard University and Bell Labs in the US observed the e/4 quasiparticles in two-dimensional electron gases in quantum-well structures made of gallium arsenide and aluminium gallium arsenide. The experiments were

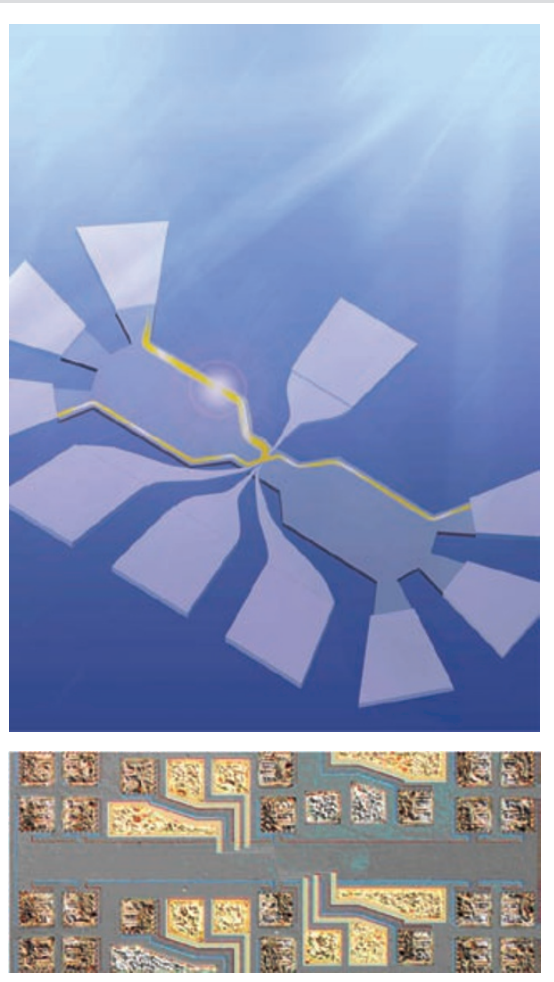

carried out at temperatures as low as $10 \mathrm{mK}$ and magnetic fields of around 5 Tesla. Under these conditions, electric currents flow along the edges of the sample rather than through the bulk.

The artist's impression (top) shows the Weizmann experiment: an electric current (yellow) enters from a source electrode on the upper left and is split in two by the quantum point contact at the centre of the device. Some of the current is transmitted to the electrode on the right, while the rest is reflected towards the left. Dolev and colleagues measure the shot noise in these currents as a function of the input current and find that their data is best explained by quasiparticles with a charge of $e / 4$ (Nature 452, 829-834; 2008).

The MIT-Harvard-Bell Labs team measures tunnelling currents through a quantum point contact in a similar setup (shown here in an optical micrograph) as a function of temperature, and again finds evidence for $e / 4$ quasiparticles. The US team also measured the strength of the interactions between the quasiparticles (Science doi:10.1026/ science.1157560; 2008).

The new quasiparticles are expected to obey an unusual type of quantum statistics called non-Abelian statistics, although this has yet to be confirmed experimentally. Most particles obey Abelian statistics: in other words, when two identical particles swap places with each other, their combined wavefunction either does not change (if they are bosons), or it gains an additional phase of $\pi$ (fermions); however, the ground state of the system is unique.

Non-Abelian systems, on the other hand, can have more than one ground state. This means that swapping two $e / 4$ quasiparticles could change the system from one ground state to a completely different ground state. A number of theorists have predicted that this property could be exploited in quantum information devices.

Peter Rodgers 